\title{
Pixel Power - Visual Methodology as a Constructive Element of a Suite of Methodologies in an Exploration of the Impacts of Cultural Elements of Irish Families on Education and Schooling in Ireland
}

\author{
Melanie Ní Dhuinn, Andrew Loxley \\ Trinity College Dublin, Ireland
}

\begin{abstract}
Discussion of methods of investigation in social science has generated considerable discourse around objectivity, knowledge status derived from various data collection instruments and the social context within which the data is situated. Ethnographers have suggested that social scientists are likely to exhibit greater confidence in their findings when these are derived from more than one method of investigation. The use of triangulation in research methodology enhances the researcher's claims for validity in affording mutual confirmation of conclusions from different methodological approaches. Visual methodology as a research method offers a methodological approach which enhances understanding and interpretation of research questions in an individual capacity or as an augmented method. When coupled with the traditional data collection instruments of questionnaires, surveys, observations, focus groups and interviews visual methodology offers a legitimate and exciting extra edge that evokes deeper elements that extend the vista of empirical research. Underpinned by the work of Bourdieu on family and sociological cultures and Bronfenbrenner on ecological development this paper explores a myriad of cultures in families and in a school using a mixed methods approach including visual methodologies to analyze and interpret the impact of family cultures on educational outcomes.
\end{abstract}

\section{Introduction}

There are many sociological arguments that have dominated and suffused the notion of family influence and the effect of family structures on children's educational and occupational success. It has been generally recognized [1], [13], [18] that families are critical in promoting educational attainment among children and are pivotal actors in the development of children's cognitive, emotional and social development [16]. From an Irish perspective however, emphases within the literature have focused on educational failure and family disadvantage, parental involvement and school effectiveness and exploration of the gendered, classed and "raced" nature of parental involvement [11]. This piece of research identifies social and cultural capital within Irish families and its influence on the educational outcomes of a selection of Irish students from various socio-economic backgrounds attending a co-educational secondary school. The research employed a variety of methodological instruments to elicit both qualitative and quantitative data which were utilized to describe the many family and school cultures that pervade the social context of families and schooling in Ireland while affording an opportunity to investigate the sometimes jarred but interdependent social context in which these cultures exist. Informed by methodological literature [ 12] that indicates that qualitative methods that rely on self reporting of thoughts, feelings and experiences of beliefs face the dilemma that internal states are known only by the individual whose prerogative it is whether or not to share them with others and also the fact that such methods of data collection are embedded in social contexts and subject to social manipulation the authors endeavored to consolidate the data by adding another methodological layer of visual methodology in an effort to enhance and develop the primary data collected through questionnaires, focus groups and interviews with parents, students, teachers and school management. Taking an empirical approach to the collection of visual data the authors gathered a combination of researcher generated and participant generated images that were used to further analyse and explore the research question. Researcher generated images included photos of the school buildings, grounds and immediate surrounding areas while participant generated images included cultural inventories created by students of their homes which included selected personal spaces of significance to the students. Students were supplied with a digital camera with an SD memory card and a shooting script and were requested to capture images of spaces within their homes that they regarded as 
significant in their lives and in the lives of their families. Students were interviewed as part of a follow up focus group and were asked about the construction of the cultural inventories depicting their homes and their selected spaces. Researcher generated images were analysed in terms of spaces within in the school buildings and grounds and the interpretation of these spaces by students and teaching staff in the school.

\section{Literature Review}

Bronfenbrenner and Ceci [6] suggest that adolescent's outcomes are related to proximal processes, which are enduring forms of interaction occurring in immediate settings such as families and schools and also distal contexts in which immediate settings are embedded. They also contend that the influences of the proximal processes on outcomes are more powerful than the influences of distal contexts [6]. Bourdieu [4], [5] alludes to the production of "habitus" whereby a system of dispositions such as aspirations arbitrates between structures and practice. Bourdieu [4] describes an inculcation effect exerted directly by families and schools on adolescents or by student's individual social backgrounds, but he also proposes a second effect arising from individual's predispositions such as the accumulation of academic capital which may cause them to resist "the forces of the field with their specific inertia that is, their properties, which may exist in the embodied form, as dispositions or in objectified form in goods, qualifications, etc.” [4].Evidence within the literature [1], [3], [4], [5], [6], indicates that students with more "valuable" social and cultural capital attain greater educational outcomes that their otherwise comparable peers with less "valuable" social and cultural capital. These theoretical arguments prompted the authors to construct a case study model to examine and evaluate the influences of social and cultural capital on student's educational outcomes while attempting to advance the description of the process whereby social and cultural resources may or may not convert favorably into educational advantages. Critical to the construction of any case study is careful consideration of useful and informative methodologies. The approach of using triangulation in research methodologies affords an opportunity to cross check findings [2] Laws argues that the key to triangulation is to see the same thing from different perspectives and therefore be able to confirm or challenge findings from one method with those of another [14].She refers to "mismatch" and "conflict" and the potential for very different accounts of similar phenomena. Mismatches should be analyzed to make sense of them [14]. Data collection instruments should be critically appraised to assess reliability and validity. Sapsford and Jupp [19] argue that what has to be established is whether data measures or characterizes what the authors claim and that interpretations do follow from the data .They continue to argue that the structure of a piece of research determines the conclusions that can be drawn from it and also what should not be drawn from it. Despite being viewed by some as a peripheral method the use of visual methodologies in both social and educational research has proceeded steadily to become a recognized and extensively utilized framework with reputable protocols for undertaking this genus of empirical work. Prosser and Loxley [17] propose three main modes of visual data construction: researcher generated- images which are constructed by a researcher specifically for a study, researcher found- images as well as artifacts which have been found or "discovered" which are considered to be relevant to the study. There is usually little known about the context of the creation of these images and participant generated-images or artifacts which are created by the research participants specifically for the study they are involved in. Additionally these can include images which have been found or "discovered" by a participant which are considered relevant for the study. Urry[20] argues that with a few notable exceptions most sociological research has been aspatial. Sociological research has tended to view space as a "backdrop" or "container" for human action rather than treat it as a critical "ingredient" in the social nexus. Loxley, O Leary and Minton in their article [15] refer to Bronfenbrenner's ecological and ecosystemic theories of child and adolescent development as being influential in representing space as a context in which social relationships are formed and impact on the developing self and how the internalized self relates to the larger environments surrounding them, they caution however that psychologists have also proceeded aspatially in the same manner as their sociological colleagues in the construction of sociological research and theorizing. Bourdieu asserts that we structure space as knowing and active agents and that in turn space structures us in that it acts as a constraint, imposing boundaries on our ideas and actions [4]. Foucault [8] expresses space as an articulation of knowledge and power which can be productive and oppressive. Halford [11] highlights the characteristic that schools are work places for both children and adults, however as Burke and Grosvener [7] argue "like other buildings, schools are the products of social behavior. They should not be viewed merely as a capsule, but as designed spaces that in their materiality, project a system of values".

\section{Methodology}

Impelled by the central research question the study investigated the influence of family social and 
cultural capital on educational outcomes by exploring the rudimentary daily activities, interactions, norms and mores of the selected families through a series of empirical verbal and visual ethnographic techniques. Subsidiary research questions include an examination of levels of parental participation in the Irish education system, the education processes through which Irish educational patterns are created and reproduced, how family social and cultural capital influences the process through which Irish parents participate in their children's education and why children from families experiencing similar social and economic circumstances attain different educational outcomes. Data was constructed through an exploratory mixed methods approach which combined both quantitative and qualitative data via questionnaires to parents $(n=$ 567), teaching staff $(\mathrm{n}=63)$ and school management $(n=10)$, focus group interviews with students $(n=4)$, and semi-structured interviews with parents $(n=25)$, parents and students $(n=25)$, school management and teaching staff $(n=10)$. Two sets of questionnaires were constructed, one to teaching staff in the research school and one to parents of children attending the research school. Questionnaires were distributed to teaching staff following a research presentation to all staff as part of a staff development day and parental questionnaires were distributed via form teachers to students in school. Staff questionnaires were returned to the authors via a collection point in the staff room and form teachers collected parental questionnaires on two nominated dates at morning assembly time in school. The research school cooperated in all aspects of data collection in their communication to parents and staff, promoting the research as a positive and informative piece of work that was mutually beneficial for all parties. Parents and staff were encouraged to participate in the research but it was emphasized that participation was voluntary and at the discretion of individual staff and families. Both parents and staff were afforded an opportunity to participate further in the research by indicating their willingness for further participation in a section at the end of each questionnaire. The parent questionnaire offered three options for further participation:; participation in a parent interview, participation in a student focus group or participation in both. Staff questionnaires offered the option of participation in a semi structured interview. Completed questionnaires were collated and analyzed using SPSS and a qualitative coding system. Participants who had indicated a willingness to participate further in the research was then included in the next phase of semi structured interviews for parents and staff and focus group interviews for students. Interviews (40 minutes in duration) with staff and management took place initially in the school with all interviews audio recorded and transcribed for analysis. Student focus groups were then held with students following acquisition of consent from parents for students to participate in the research. Focus groups lasted 45 minutes and were conducted in a room at the school. All focus groups were transcribed and coded for analysis. The interview/focus group room was away from the main school area and therefore was very suitable for the task in hand with no interruptions. Following the semi- structured interviews and the focus groups a small number $(n=4)$ of families indicated their willingness to participate in the construction of visual cultural inventories as part of the research. Students were supplied with a digital camera, a SD memory card and a shooting script and asked to visually capture spaces in their family homes (inside and outside) that represented significant spaces for them. The authors captured the visual cultural inventory of the school buildings and surroundings, grounds and approaches using a digital camera. The authors met with the students in a focus group to discuss and analyze the images they had constructed and also to discuss the visual cultural inventory of the school buildings and grounds.

\section{Discussion}

This research is an evaluation of the influence of family social and cultural capital on the educational outcomes of a sub-selected cohort of students $(n=25)$, from a base of 567 families from a coeducational secondary school in the South East of Ireland. The total school enrolment numbers 789 students with a staff complement of 63 teachers. Of the initial 567 questionnaires distributed to families 389 were returned. 40 of 63 staff questionnaires were returned. 4 families agreed to participate in the collation of visual cultural inventories. The combination of data sourced through questionnaires, focus groups and semi structured interviews provided a rich insight into participant's affective dispositions and viewpoints. This data was positively enhanced by the addition of the visual cultural inventories constructed by students and analysed through a second focus group. Questionnaires to parents elicited information about: socio-economic status, educational attainment levels, occupation, views of their own education, children's education, participation as a parent in their children's education and family life and education. Staff questionnaires elicited information about educational qualifications, institutions of study, subjects studied, subjects taught, number of hours teaching per week, additional responsibilities within the school, subject teams and professional development. Student focus groups focused on likes and dislikes about school. Students articulated various likes and dislikes and openly discussed their memories of beginning at the school, their thoughts about their current situation 
and their expectations for their futures. Likes and dislikes included discussions about the school uniform and dress code.

“The $v$ neck jumper was easier to manage, I don't like the round neck, it looks awful. And we should be able to have our hair whatever way we want, its only hair...they're too strict about the hair"

Students also talked about their initial time at the school:

"I was really afraid for the first few weeks, I didn't know anyone and I was nervous"

"I was looking forward to it, I had heard so much from my sisters that I kinda knew what to expect."

Others referred to their choice to attend the school. When asked who made the choice for them to attend the school one of the students replied:

"I did I suppose, but my Mam and Dad wanted me to come here too. My sisters went here and they knew the school and they were happy enough with it..."

Discussions followed about their goals for the future and the conversations they had at home with parents, grandparents and siblings about going to college and future careers. Students also referred to the types of activities that did in class and out of class time.

"In class we do our work, the teacher comes in and we just do the subjects and stuff, its either 35 minutes or 40 minutes depending on the day or the time and then the bell goes and we have the next class. Sometimes we stay in the room or sometimes we move to another room if its Biology or Art or PE or Construction, its all pretty quick though cos you're not allowed to be late or anything or you're in trouble."

Students were very vocal about their space in the school, they each had designated areas in which to gather and socialize but some of them were unhappy about the fact that they couldn't go from one area to the other to meet friends form different year groups as the areas were clearly demarcated and bounded by year group status. Even though the spaces were not extensive they did feel an ownership of the space as theirs and were very defensive of it.

"In the year group areas, all the year groups have their own areas and we have to stay in those areas, we cant go into the $1^{\text {st }}$ year areas or we're in trouble, sometimes we can go down to the pitches or that and sometimes we just hang around the classrooms"
Students were asked to talk about their worries and what they were looking forward to.

"Exams, this year and next year in the Leavin, I have to do well this year so I won't have so much to do next year"

"When the Leavin is over I will miss my friends, and I won't see them all again, I have a good few friends but I won't have them when I leave"

Participant visual cultural inventories constructed by students revealed that they valued their kitchens, bedrooms (shared or single), outside spaces and TV /Living room as spaces they felt were significant and indicative of their lives and their participation in family lives.

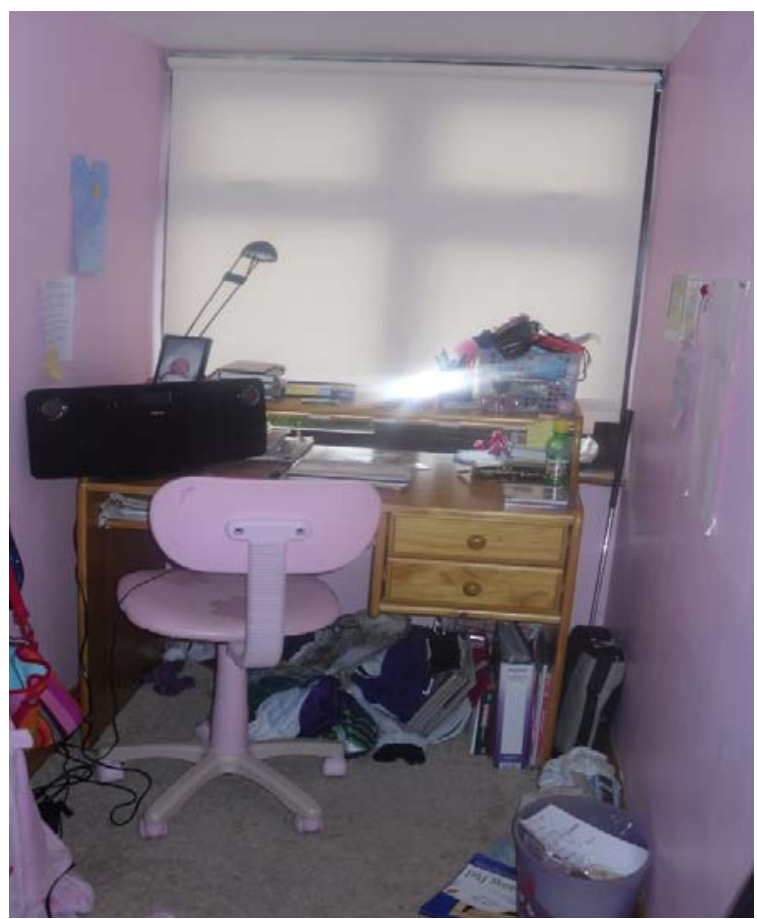

Figure 1 


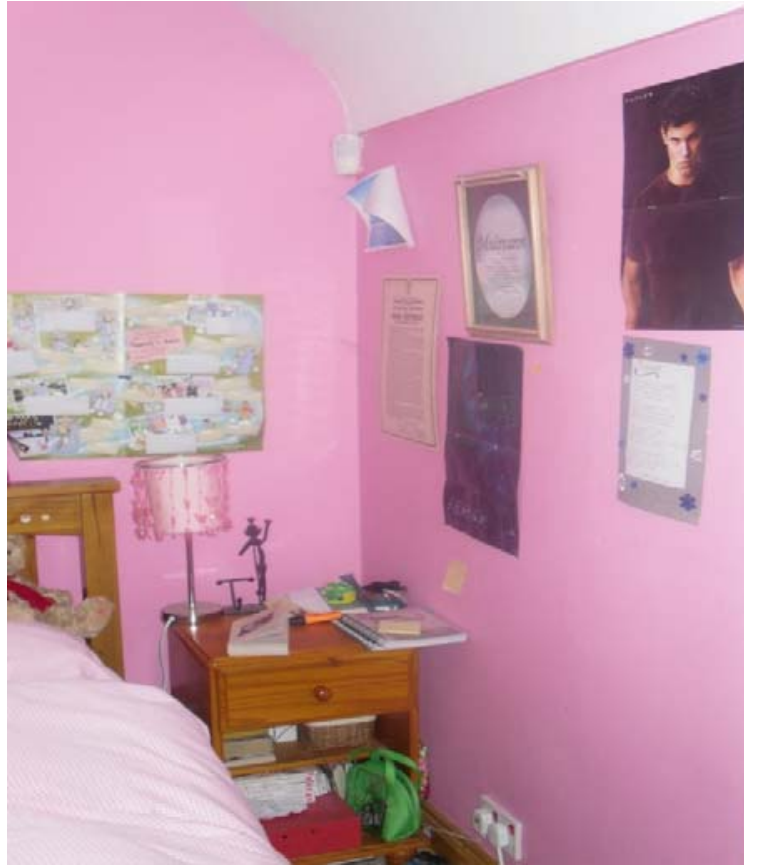

Figure 2

Researcher generated visual cultural inventories of the school indicated bounded, institutional type, ordered spaces where architecture, furniture and fittings all indicated compliance and production of social order.

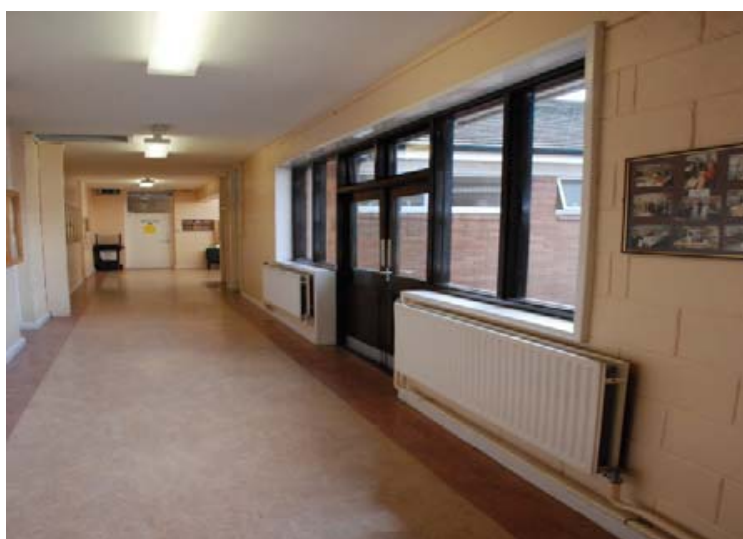

Figure 3

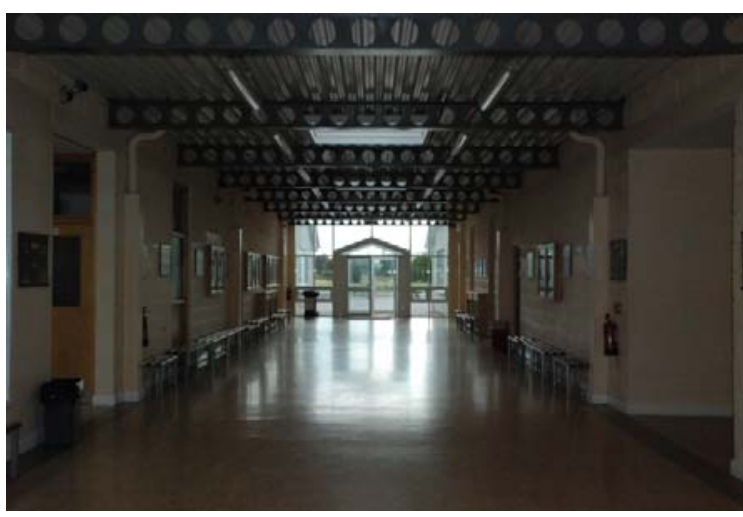

Figure 4

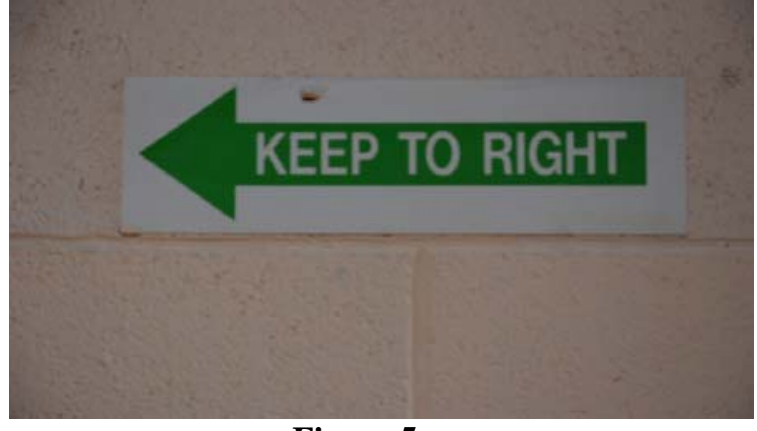

Figure 5

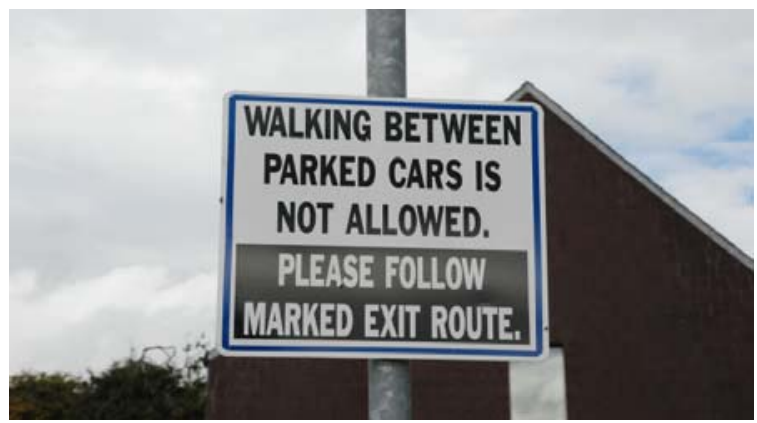

Figure 6

The visual cultural inventories of the school and the homes are noticeably different in terms of the ordered bounded spaces in the school projecting a specific system of values in comparison with the free, unbounded, somewhat unordered living spaces of the homes also projecting family values demonstrates two separate cultures. These two cultures are however inextricably linked as students have to manage moving from the home spaces of freedom and expression to the school culture of rules and regulations. Central to this link is however the innate family culture and associated values that are moulded, mentored and modelled in the school setting. It is the merge of both cultures that coincide to further a single culture promoting educational attainment and progression of families and thereafter reproducing the social order.

\section{Conclusion}

While acknowledging the complex and contested nature of the concept of social and cultural capital and notwithstanding the fact that the transfer and reproduction of social and cultural capital is not always spontaneous and continuous, but frequently intermittent, jagged and contingent on the social field from which it originates the authors have constructed a comprehensive ethnographic snapshot of a previously unexplored canvas in Irish secondary schooling unveiling the influence of family social and cultural capital on Irish secondary students from a variety of socio-economic backgrounds. 


\section{References}

[1] Archer, L.; Hutchings M.; Ross, A.; Leathwood, C.; Gilchrist R.; Phillips D (2003). Higher education and social class: issues of exclusion and inclusion. London : Routledge Falmer.

[2] Bell, J (2005) Doing Your Research Project A guide for first-time researchers in education, health and social science $4^{\text {th }}$ edition Open University Press England

[3] Bourdieu, P., and Passeron, J.-C. (1977), Reproduction In Education, Society and Culture. Tr. By R. Nice. Beverly Hills, CA: Sage.

[4] Bourdieu, P. (1984a). Distinction: A Social Critique of the Judgement of Taste. Cambridge, MA: Harvard University Press.

[5] Bourdieu, P (1988). Homo academicus. Cambridge, U.K.: Polity Press.

[6] Bronfenbrenner, U \& Ceci, S J. (1994). Nature-nurture reconceptualization in development perspective: A bioecological model. Psychological Review, 101, 568-586.

[7] Burke, C \& Grosvenor, I. (2003) The school I'd like. Children and Young People's reflections on an education for the $21^{\text {st }}$ century London: Routledge

[8] Foucault, M (1991) Truth and Power. In P.Rainbow (Ed), The Foucault Reader: Harmondsworth: Penguin Books

[9] Grenfell, M (2008). Pierre Bourdieu Key Concepts, Acumen.

[10] Halford, S. (2008). The Sociology of space, work and organisation: From fragments to spatial theory. Social Compass, 2(3), 925-943

[11] Hanafin, J; Lynch A, (2002) Peripheral Voices: Parental Involvement, social Class, and educational Disadvantage British Journal of Sociology, Vol 23, No 1pp 35-49.

[12] Hollander, J.A.; (2004) The Social Contexts of Focus Groups Journal of Contemporary Ethnography, Vol 33, No 5, pp 602-637.

[13]Israel et al (2001) "The Influence of Family and Community Social Capital on Educational Achievement” Rural Sociology pp 43-68

[14]Laws, S. (2003) in Bell, J. (2005) Doing Your Research Project A guide for first-time researchers in education, health and social science $4^{\text {th }}$ edition Open University Press England

[15]Loxley, A.; O Leary, B. ; Minton, S.J. Space Makers or Space Cadets? Exploring children's perceptions of space and place in the context of a Dublin primary school. Educational \& Child Psychology Vol 28, No1 British Psychological Society

[16]Phillips, S \& Eustace, A (2008) Family Involvement in Education, PLANET-The Partnerships Network \& POBAL- Supporting Communities, Dublin.

[17] Prosser, J.\& Loxley, A (2008). Introducing Visual methodology.Economic and Social Science Research Council: National Centre for Research Methods, Swindon.

[18]Ryan, B A; Adams, G, Gullotta, T; Weissberg, R; Hampton, R; (1995)The Family School Connection Volume 2 Issues in Children's and Families Lives Sage Publications London.

[19] Sapsford, R \& Jupp, V. (1996) Data Collection and Analysis. London: Sage

[20]Urry, J. (1995). Sociology of time and space. In B. Turner (Ed), Blackwell companion to social theory. Oxford: Blackwell. 